\title{
Therapeutic Hypothermia after Decompressive Craniectomy in Malignant Cerebral Infarction
}

\author{
Jun Young Chang, M.D., Jeong-Ho Hong, M.D. , Jin-Heon Jeong, M.D., Sung-Jin Nam, M.D., Ji-Hwan Jang, M.D. ${ }^{\dagger}$, \\ Jae Seung Bang, M.D. ${ }^{\dagger}$, and Moon-Ku Han, M.D. ${ }^{\ddagger}$ \\ Department of Interdisciplinary Care Medicine, Seoul National University Bundang Hospital, Seongnam; *Department of Neurology, Keimyung University \\ Dongsan Medical Center, Daegu; Departments of ${ }^{\dagger}$ Neurosurgery, and ${ }^{\ddagger}$ Neurology, Seoul National University Bundang Hospital, Seongnam, Korea
}

Decompressive hemicraniectomy followed by subsequent therapeutic hypothermia can reduce mortality in patients with malignant cerebral infarction without significantly increasing risk. We report three cases of malignant cerebral infarction treated with hemicraniectomy followed by hypothermia. Case 1 received elective decompressive surgery and hypothermia. Case 2 developed subsequent cerebral infarction with uncal herniation. Therefore, emergent decompressive surgery and hypothermia was performed in this case. Despite surgery and hyperosmolar therapy, case 3 received hypothermia treatment for refractory increased intracranial pressure. All patients survived with a score of 4 or 5 on the modified Rankin scale. Therefore, we suggest that application of hypothermia after hemicraniectomy is safe and feasible. Several possible modifications can be made to improve the management strategy in order to increase the benefits of hypothermia treatment.

Key Words: brain edema; cerebral infarction; decompressive craniectomy; hypothermia.

Malignant cerebral infarction consists of $3 \%$ to $10 \%$ of supratentorial cerebral infarctions and $80 \%$ of the patients die without proper treatment.[1,2] Early decompressive surgery prior to herniation is known to reduce 1- and 6-month mortality to 4.8 and $19.1 \%$, respectively.[3] Moderate hypothermia is also a useful therapeutic option for space-occupying cerebral edema, significantly lowering elevated intracranial pressure (ICP) and protecting from further neuronal damage.[4] Moreover, therapeutic hypothermia after the hemicraniectomy has an additional effect on decreasing mortality and functional disability without increasing the occurrence of adverse events.[5] We have studied three patients with malignant cerebral infarction who underwent

Received on February 4, 2014

Revised on April 2, 2014

Accepted on April 16, 2014

Correspondence to: Moon-Ku Han, Department of Neurology, Seoul

National University Bundang Hospital, 82 Gumi-ro 173beon-gil,

Bundang-gu, Seongnam 463-707, Korea

Tel: +81-31-787-7464, Fax: +82-31-787-4059

E-mail: mkhan@snubh.org decompressive hemicraniectomy and subsequent hypothermia without serious complications.

\section{CASE REPORT}

\section{1) Case 1}

A 26-year-old man presented to the emergency room (ER) with right hemiparesis, global aphasia, and drowsiness with a history of pulmonary embolism and exertional dyspnea for a month. He was brought to a nearby tertiary hospital and initial brain magnetic resonance imaging (MRI) revealed large acute infarction in the left middle cerebral artery (MCA) with left proximal internal carotid artery (ICA) occlusion. After recanalization therapy had failed, the patient was transferred to the neuroICU in our hospital. Upon arrival at the neuroICU, initial National Institutes of Health Stroke Scale (NIHSS) score of the patient was 22. As a development of malignant cerebral edema was predicted, the patient received an elective hemicraniectomy 14 hours after symptom onset and therapeutic hypothermia was

cc This is an Open Access article distributed under the terms of the Creative Commons Attribution Non-Commercial License (http://creativecommons.org/ licenses/by-nc/3.0/) which permits unrestricted non-commercial use, distribution, and reproduction in any medium, provided the original work is properly cited. 

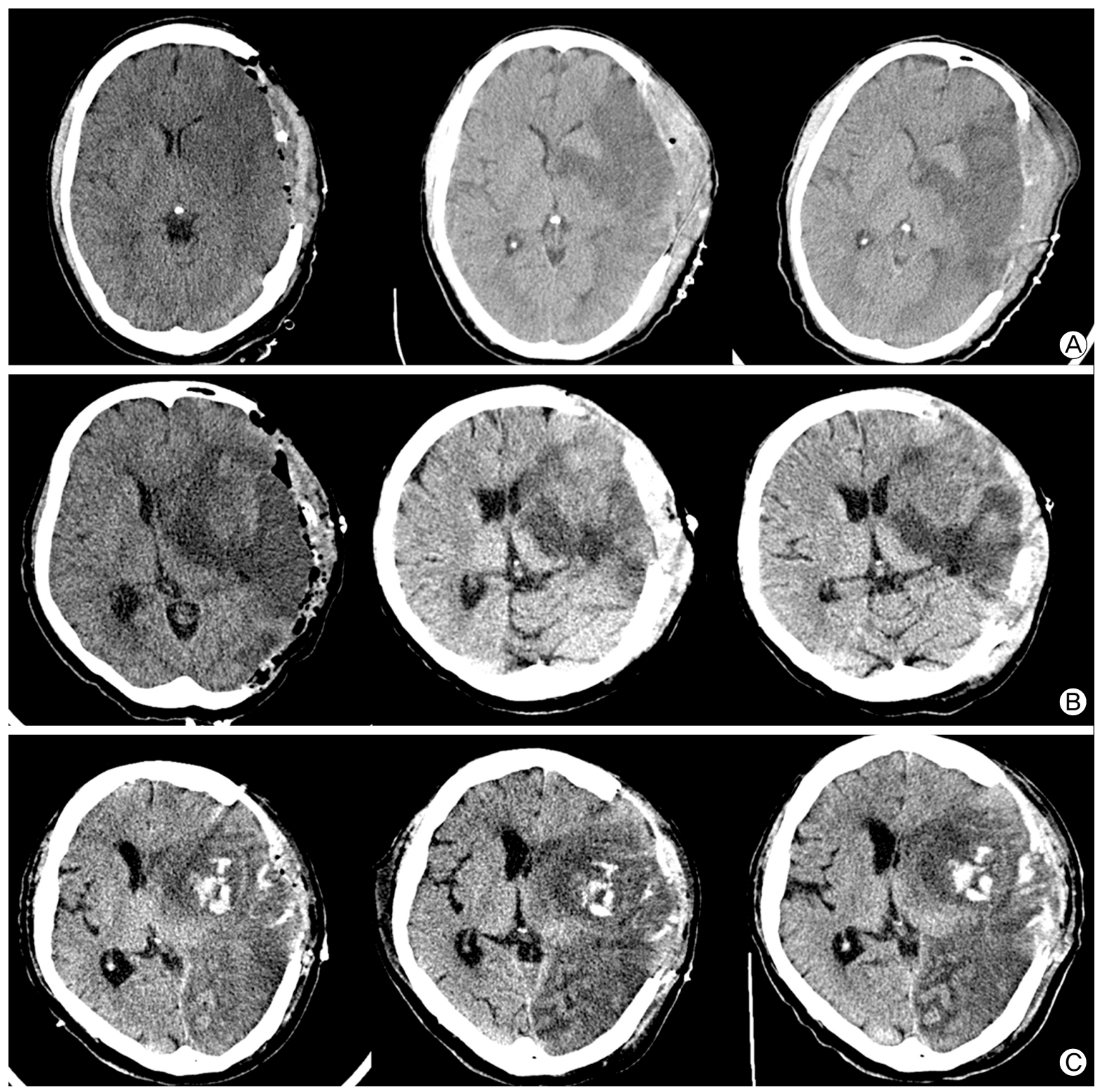

Fig. 1. Serial brain CT of the patients. (A) Case 1: Brain CT was checked at postoperative day 0, 4, 7. Midline shifting was minimal and the cerebral edema ceased to progress between day 4 and day 7. (B) Case 2: Brain CT was performed at postoperative day 1, 4 and 7. Midline shifting and obstructive hydrocephalus were evident at day 1. Cerebral edema was gradually decreased during hypothermia treatment. (C) Case 3: Brain CT was evaluated at postoperative day 0, 4 and 6. Large hemispheric infarction was accompanied by hemorrhagic transformation at day 0 . Despite the infarct area became more evident, the degree of midline shifting did not increase till day 8 .

subsequently applied. During that period, the progression of cerebral edema and midline shifting was minimal on serial brain computed tomography (CT) images (Fig. 1a). After verifying that cerebral edema ceased to progress, rewarming was initiated 6 days following hypothermia. Due to hypotension caused by hypothermia and anti-shivering medications, inotropic agents were administered for 7 days. Sinus bradycardia (with a heart rate of 60 beats/min or less) occurred during the first 3 days after applying hypothermia but recovered without specific management.
Mechanical ventilation was maintained for 9 days and discontinued after pneumonia was resolved. The patient was transferred to the recovery ward at 21 days after admission. The patient survived and was discharged with mild improvement of NIHSS total score of 16. Modified Rankin scale (MRS) score at discharge was the same as the initial score of 5. The laboratory results for hypercoagulable states including anti-phospholipid antibodies and prothrombin gene mutation were all negative. 


\section{2) Case 2}

A 50-year-old woman with history of paroxysmal atrial fibrillation and hypertension was found after having fallen in her bathroom with an altered mental status. In the ER, she presented with drowsiness, was unable to speak and had decreased withdrawal response to pain stimuli on the right side implying right hemiplegia or hypesthesia. Initial NIHSS was 22. Diffusionweighted image revealed a superior division of the left MCA, anterior cerebral artery (ACA) territorial infarction with massive hemorrhagic transformation and midline shifting $(4.3 \mathrm{~mm})$. Based on the decision of the attending neurologist, hyperosmolar therapy was initiated instead of surgery. The midline shifting was increased to $8.2 \mathrm{~mm}$ on the fourth day and ceased to progress on the seventh day without obvious neurologic deterioration. However, at day 10 , her mental status became stuporous, left pupil was dilated and nonreactive to light. Brain CT revealed a newly developed inferior division of the left MCA infarction with uncal herniation. Emergent decompressive hemicraniectomy was performed and moderate hypothermia was induced after the operation. Midline shifting and obstructive hydrocephalus gradually decreased during the treatment (Fig. 1b). Hypothermia was maintained for 2 days and slowly rewarmed for $16 \mathrm{~h}$. Normothermia was continued for 2 days. Due to hypotension at the beginning of the hypothermia therapy, continuous inotropic agents were infused for 4 days to maintain mean arterial pressure of 90 to $110 \mathrm{mmHg}$. Transient slow ventricular response of atrial fibrillation with a heart rate less than 40 beats/min was observed, but recovered immediately without medications. As the mental status of the patient improved to near alert, pupillary reflex was restored and midline shifting decreased to $2 \mathrm{~mm}$ in the brain CT image, we decided to move her to the general ward on the eleventh day after neuroICU readmission. The NIHSS score at discharge was 22 and MRS score was 5. The MRS score 3 months later did not improve.

\section{3) Case 3}

A 76-year-old man with history of atrial fibrillation and Parkinsonism developed an acute right-sided hemiparesis and global aphasia (NIHSS score of 23). In the ER, brain MRI revealed a left MCA infarction with distal ICA occlusion. The occluded vessel was completely recanalized after thrombolysis. However, the patient became stuporous and brain CT revealed aggravated cerebral edema after the procedure. We decided to perform a decompressive hemicraniectomy at 17 hours after symptom onset. Despite the surgery and hyperosmolar therapy, the left pupil became dilated, nonreactive and midline shift pro- gressed to $7 \mathrm{~mm}$ at follow-up CT imaging (Fig. 1c). Moderate hypothermia was induced at 24 hours after symptom onset, maintained for 7 days and slowly rewarmed to normothermia for 24 hours. After maintaining normothermia for 30 hours, hypothermia was discontinued. Bradycardia with a rate below 40 beats/min was sustained for 3 days and was resolved without treatment. The methicillin-resistant Streptococcus aureus associated cellulitis at the tracheostomy site was treated with an intravenous vancomycin. Cerebral edema gradually improved and the patient was able to open his eyes in response to painful stimuli. The patient stayed at the neuroICU for 23 days. The NIHSS and MRS scores at discharge were 21 and 4, respectively.

The characteristics of the patients are summarized in Table 1.

\section{Decompressive craniectomy}

A question mark-shaped skin incision was made $1 \mathrm{~cm}$ ahead of a tragus. A large craniectomy including frontal, temporal and parietal bones was performed. Dura was opened in semicircular and radial shapes to retain a sufficient space for the swollen brain. Duroplasty was done using lyophilized bovine pericardium (Lyoplant ${ }^{\circledR}, \mathrm{B}$. Braun). The infarcted area of the brain was not removed. The bone flap was stored in $100 \%$ ethanol until performing cranioplasty, usually at least 6 weeks after craniectomy.

\section{Therapeutic hypothermia}

Moderate hypothermia $\left(33.5^{\circ} \mathrm{C}\right)$ achieved by a surface cooling device (Arctic Sun ${ }^{\circledR}$, Medivance, USA) was started after the operation. Surface cooling pads were attached at the trunk, back, and both thighs and core temperature was monitored via an esophageal probe. One liter of cold saline $\left(4^{\circ} \mathrm{C}\right)$ was administered for 30 min to reach the target temperature as soon as possible. To minimize shivering, warming blankets were routinely used for all hypothermic patients. In case shivering was not well controlled with surface warming, intravenous meperidine (50-100 mg bolus followed by $12.5-50 \mathrm{mg} / \mathrm{h}$ ), dexmedetomidine (loading dose $1 \mu \mathrm{g} / \mathrm{kg}$ over $10 \mathrm{~min}$ followed by an infusion of $0.3-1.5$ $\left.\mu \mathrm{g} / \mathrm{kg}^{-1} / \mathrm{h}^{-1}\right)$, fentanyl (25-75 $\mu \mathrm{g}$ bolus dose followed by $50-200$ $\mu \mathrm{g} / \mathrm{h})$, and magnesium $(1 \mathrm{~g} / \mathrm{h}$ and titrate to serum magnesium of $3 \mathrm{mmol} / \mathrm{L}$ ) were used in that sequence. The duration of hypothermia maintenance was determined by clinical decision of the physician. Due to a risk of rebound cerebral edema, rewarming was slowly conducted at a rate of $0.1^{\circ} \mathrm{C} / \mathrm{h}$. The latter two cases retained normothermia for a certain period after rewarming was completed. During the treatment, the occurrence of possible side effects such as hypo- or hypertension, bradycardia, arrhythmia, 
Table 1. Characteristics and outcomes of the three patients underwent decompressive hemicraniectomy followed by moderate hypothermia

\begin{tabular}{|c|c|c|c|}
\hline & Case 1 & Case 2 & Case 3 \\
\hline Location/Mechanism & Lt. MCA/ Undetermined & Lt. ACA, MCA / CE & Lt. MCA/ CE \\
\hline Initial NIHSS/MRS & $22 / 5$ & $22 / 5$ & $23 / 5$ \\
\hline Reason for additional hypothermia & Elective & $\begin{array}{l}\text { Urgent IICP lowering due to } \\
\text { herniation }\end{array}$ & $\begin{array}{l}\text { Refractory IICP despite surgery } \\
\text { and hyperosmolar therapy }\end{array}$ \\
\hline Time to surgery/Time to hypothermia (h) & $14 / 20$ & $10 / 48$ & $17 / 29$ \\
\hline $\begin{array}{l}\text { Target temperature }\left({ }^{\circ} \mathrm{C}\right) / \text { Duration of hypothermia }(\mathrm{d}) \\
\text { /Duration of rewarming }(\mathrm{h})\end{array}$ & $33.5 / 6 / 30$ & $33.5 / 2 / 16$ & $33.5 / 7 / 24$ \\
\hline Normothermia maintenance/Duration (h) & Not done & Yes/48 & Yes/30 \\
\hline Maximal midline shifting $(\mathrm{mm})$ & 2 & 8 & 7 \\
\hline The length of ICU stay (d) & 21 & 11 & 23 \\
\hline The length of mechanical ventilation (d) & 9 & 10 & 21 \\
\hline The duration of Mannitol /Inotropics infusion (d) & $6 / 7$ & $9 / 4$ & $15 / 5$ \\
\hline Mortality/3 month MRS & Survived/5 & Survived/5 & Survived/4 \\
\hline Adverse effects & Hypotension, pneumonia & Hypotension, phlebitis & $\begin{array}{l}\text { Hypotension, bradycardia } \\
(\mathrm{HR}<40) \text {, cellulitis }\end{array}$ \\
\hline
\end{tabular}

Lt: left; MCA: middle cerebral artery; ACA: anterior cerebral artery; CE: cardioembolism; NIHSS: National Institutes of Health Stroke Scale; MRS: Modified Rankin scale; IICP: increased intracranial pressure; HR: heart rate.

electrolyte imbalance, coagulopathy, and infection were closely monitored and managed.

\section{The protocol of intensive care management during therapeutic hypothermia}

All patients who underwent decompressive hemicraniectomy were sedated using midazolam, remifentanyl, and cisatracurium aimed at a Ramsay score of 4 or 5 . We monitored arterial blood pressure directly through the radial artery. Fluid infusion and inotropic medication were adjusted to maintain mean arterial pressure (MAP) in a range of $90-110 \mathrm{mmHg}$, cerebral perfusion pressure (CPP) over $70 \mathrm{mmHg}$, and central venous pressure from $8-12 \mathrm{cmH}_{2} \mathrm{O}$. The position of the patients was maintained with their head elevated about $30^{\circ}$. Hyperosmolar therapy was indicated when ICP exceeded $20 \mathrm{mmHg}$. Blood glucose level was kept between 120-150 mg/dl, and arterial blood $\mathrm{CO}_{2}$ between 36 and $40 \mathrm{mmHg}$. Therapeutic hypothermia was terminated if severe bleeding, intractable hypotension (MAP $<50$ $\mathrm{mmHg}$ despite fluid resuscitation and inotropic medications), or multi-system organ failure occurred.

\section{DISCUSSION}

Based on several mechanisms of neuroprotection, [6-8] the additional effect of hypothermia on MCA after hemicraniectomy has been explored and revealed improved functional outcome without significant side effects.[5] Nevertheless, the sample size of the previous study was too small to generalize the results.[5] The DEcompressive surgery Plus hypoTHermia for Space-Occupying Stroke (DEPTH-SOS) study planned as a multicenter, randomized controlled trial has just commenced with the primary outcome of mortality at day 14.[9] Though none of the patients died, the MRS of the patients at 3 months was 4 or 5 , indicating severe functional disability remained after 3 months. The addition of therapeutic hypothermia might be helpful for preventing death from the disease, but it is insufficient to improve functional outcome and thereby quality of life.

The time interval between symptom onset and starting hypothermia ranged from 20 to 48 hours in our cases. Due to a concern that adverse events such as hypotension or coagulopathy might be problematic on applying hypothermia before or during the surgery, hypothermia was applied as soon as possible after the surgery. However, intraoperative hypothermia has been used during intracranial aneurysm clipping and thoraco-abdominal aortic aneurysm surgery, which have been proven safe for reducing an ischemic insult of the brain and spinal cord induced by transient occlusion of the vessel.[10,11] According to a previous animal study, focal ischemic injury progresses more rapidly and the time window for preventing neurologic injury is narrow compared with a traumatic brain injury or global ischemia.[12] Once hypothermic therapy is chosen, the initiation should be prompt even before surgery and maintained during the procedure. The latency to reach the target temperature should also be minimized by cold saline infusion and appropriate shivering management.

A consensus on optimal cooling duration has not yet been reached for malignant cerebral infarction. In our cases, the duration of maintaining target temperature was 2, 6, and 7 days, respectively. Usually, infarct tissue swelling was maximal between the second and fifth day after stroke onset[13] and we maintained the treatment for at least 48 hours. In the patient who 
had maintained hypothermia for 2 days, the degree of maximal midline shifting was greatest, but the length of ICU stay was the least among the three. In the patient with the longest duration of hypothermia for 7 days, the length of ICU stay and mechanical ventilation was also the longest. The duration of hypothermia should be individualized by weighing the risks and benefits and be maintained at least until the cerebral edema stops increasing. The treating physician should also carefully evaluate any occurrence of neurologic deterioration after discontinuing the hypothermia treatment.

Rewarming was slowly performed after ascertaining that cerebral edema ceased to progress. We adopted a slower rate of 0.05 to $0.1^{\circ} \mathrm{C} / \mathrm{h}$, which took 16 to 30 hours. ICP elevation and CPP decrement was slow with more gradual and controlled rewarming after hypothermia.[14] When rewarming rate is not cautiously regulated, the risk of developing rebound edema increases.[15,16] However, in our cases, rebound edema did not occur. Owing to a detrimental effect of fever in cerebral ischemia,[17] we decided to retain normothermia for 30 and 48 hours in the latter two patients at risk of developing fever caused by infection.

The most frequent side effect was hypotension that occurred in all of the cases and required intravenous inotropic agents to maintain target mean arterial pressure. Whether the low blood pressure was attributed to the hypothermia, sedatives or anti-shivering medications is unclear. Severe, sustained bradycardia without intractable hypotension occurred in one patient but this patient regained normal heart rate spontaneously despite ongoing hypothermia. The slowing of metabolism, decreased left ventricular contractility and cardiac output induced by hypothermia causes hypotension and bradycardia, although symptomatic bradycardia or arrhythmia do not occur frequently at body temperatures above $30^{\circ} \mathrm{C}$.[18] As the myocardium becomes refractory to antiarrhythmic medications or defibrillation,[18] termination of the hypothermia should be considered in the presence of symptomatic bradycardia or arrhythmia. Hospital-acquired pneumonia, venous access site phlebitis, and cellulitis were reported in the cases, all of which were cured by proper management. Regardless of the treatment duration, no significant infection occurred. Recent meta-analyses confined to studies of cerebral ischemia or traumatic brain injury found that the occurrence of infection was not significantly different between hypothermia and control groups.[19] With a close monitoring in the neuroICU, we could easily detect and safely manage the treatment-related complications.

In summary, we report the treatment of three cases of malignant cerebral infarction with decompressive craniectomy and therapeutic hypothermia. Application of hypothermia after hemicraniectomy was safe and feasible. Several modifications of management strategy could possibly maximize the benefit of hypothermia. We should strive to reduce time from symptom onset to treatment initiation. Duration of cooling should be maintained until progression of edema is halted, followed by slow, controlled rewarming. Maintenance of normothermia in patients with combined infection should also be considered. Given our present findings, we are planning to conduct a randomized controlled trial comparing the beneficial effect of hemicraniectomy alone and hemicraniectomy with therapeutic hypothermia in the malignant cerebral infarction.

\section{REFERENCES}

1) Hacke W, Schwab S, Horn M, Spranger M, De Georgia M, von Kummer R: 'Malignant' middle cerebral artery territory infarction: clinical course and prognostic signs. Arch Neurol 1996; 53: 309-15.

2) Vahedi K, Hofmeijer J, Juettler E, Vicaut E, George B, Algra A, et al: Early decompressive surgery in malignant infarction of the middle cerebral artery: a pooled analysis of three randomised controlled trials. Lancet Neurol 2007; 6: 215-22.

3) Mori K, Nakao Y, Yamamoto T, Maeda M: Early external decompressive craniectomy with duroplasty improves functional recovery in patients with massive hemispheric embolic infarction: timing and indication of decompressive surgery for malignant cerebral infarction. Surg Neurol 2004; 62: 420-9.

4) Schwab S, Schwarz S, Spranger M, Keller E, Bertram M, Hacke W: Moderate hypothermia in the treatment of patients with severe middle cerebral artery infarction. Stroke 1998; 29: 2461-6.

5) Els T, Oehm E, Voigt S, Klisch J, Hetzel A, Kassubek J: Safety and therapeutical benefit of hemicraniectomy combined with mild hypothermia in comparison with hemicraniectomy alone in patients with malignant ischemic stroke. Cerebrovasc Dis 2006; 21: 79-85.

6) Chi OZ, Liu X, Weiss HR: Effects of mild hypothermia on blood-brain barrier disruption during isoflurane or pentobarbital anesthesia. Anesthesiology 2001; 95: 933-8.

7) Xu L, Yenari MA, Steinberg GK, Giffard RG: Mild hypothermia reduces apoptosis of mouse neurons in vitro early in the cascade. J Cereb Blood Flow Metab 2002; 22: 21-8.

8) Kimura A, Sakurada S, Ohkuni H, Todome Y, Kurata K: Moderate hypothermia delays proinflammatory cytokine 
production of human peripheral blood mononuclear cells. Crit Care Med 2002; 30: 1499-502.

9) Neugebauer H, Kollmar R, Niesen WD, Bosel J, Schneider $\mathrm{H}$, Hobohm $\mathrm{C}$, et al: DEcompressive surgery Plus hypoTHermia for Space-Occupying Stroke (DEPTH-SOS): a protocol of a multicenter randomized controlled clinical trial and a literature review. Int J Stroke 2013; 8: 383-7.

10) Frank SM, Parker SD, Rock P, Gorman RB, Kelly S, Beattie $\mathrm{C}$, et al: Moderate hypothermia, with partial bypass and segmental sequential repair for thoracoabdominal aortic aneurysm. J Vasc Surg 1994; 19: 687-97.

11) Hindman BJ, Todd MM, Gelb AW, Loftus CM, Craen RA, Schubert A, et al: Mild hypothermia as a protective therapy during intracranial aneurysm surgery: a randomized prospective pilot trial. Neurosurgery 1999; 44: 23-32.

12) Polderman KH: Application of therapeutic hypothermia in the ICU: opportunities and pitfalls of a promising treatment modality. Part 1: Indications and evidence. Intens Care Med 2004; 30: 556-75.

13) Cheng-Mei S, Alvord EC, Jr, Berry RG: Swelling of the brain following ischemic infarction with arterial occlusion. Arch Neurol 1959; 1: 161-77.

14) Steiner T, Friede T, Aschoff A, Schellinger PD, Schwab S,
Hacke W: Effect and feasibility of controlled rewarming after moderate hypothermia in stroke patients with malignant infarction of the middle cerebral artery. Stroke 2001; 32: 2833-5.

15) Schwab S, Georgiadis D, Berrouschot J, Schellinger PD, Graffagnino C, Mayer SA: Feasibility and safety of moderate hypothermia after massive hemispheric infarction. Stroke 2001; 32: 2033-5.

16) Hong JH, Jeong JH, Chang JY, Yeo MJ, Jeong HY, Bae HJ, et al: Repeated hypothermia for rebound cerebral edema after therapeutic hypothermia in malignant cerebral infarction. Korean J Crit Care Med 2013; 28: 221-4.

17) Hajat $C$, Hajat $S$, Sharma P: Effects of poststroke pyrexia on stroke outcome : a meta-analysis of studies in patients. Stroke 2000; 31:410-4.

18) Polderman KH: Application of therapeutic hypothermia in the ICU: opportunities and pitfalls of a promising treatment modality. Part 1: Indications and evidence. Intens Care Med 2004; 30: 556-75.

19) Geurts M, Macleod MR, Kollmar R, Kremer PH, van der Worp HB: Therapeutic hypothermia and the risk of infection: a systematic review and meta-analysis. Crit Care Med 2014; 42: 231-42. 